\title{
Panorama da Aplicação de Massive Open Online Course (MOOC) no Ensino Superior: Desafios e Possibilidades
}

Overview of the application of Massive Open Online Course (MOOC) in Higher Education:

Marcos Vinícius Mendonça Andrade ${ }^{1 *}$, Ismar Frango Silveira ${ }^{2}$

${ }^{1}$ Doutorando em Ensino de Ciências e Matemática, Universidade Cruzeiro do Sul. São Paulo - SP - Brasil. marcosvinicius@vm.uff.br

2 Doutor em EngenhariaElétrica, Professor Titular, Universidade Cruzeiro do Sul. São Paulo - SP Brasil. ismarfrango@gmail.com

\section{Resumo}

Este trabalho aborda a aplicação dos Massive Open On-line Courses - MOOC - no contexto do Ensino Superior. Traz um breve histórico, características e principais tipos de $\mathrm{MOOC}$, bem como os relaciona com o movimento da Educação Aberta. Demonstra através de mapeamento sistemático da literatura um panorama dos estudos publicados na área, tendo como recorte temporal o período entre 2011 e 2015. Os resultados obtidos evidenciam que a aplicação dos MOOC no Ensino Superior é apontada como tema emergente e descortina uma série de potencialidades e desafios, exigindo uma nova postura das instituições de ensino superior. Ressalta que os estudos nessa temática ainda são incipientes.

Palavras-chave:: MOOC, Massive Online Open Course, Ensino Superior, Educação Aberta, Tecnologias Emergentes. 


\section{Overview of the application of Massive Open Online Course (MOOC) in Higher Education: Challenges and Opportunities}

\section{Abstract}

This paper proposes to identify application of Massive Open Online Courses MOOC - in higher education context. It provides a brief history, features and main types of MOOC as well as relates to the movement of Open Education. Demonstrates through systematic mapping of literature an overview of the studies published in the field, with the time frame the period between 2011 and 2015. The results show that the application of MOOC in higher education is seen as an emerging theme and opens up a number of potential and challenges requiring a new approach of higher education institutions. It is evident although the studies on this topic are still incipient.

Keywords: MOOC, Massive Online Open Course, Higher education, Open Education, Emerging Technologies

\section{Introdução}

Com a ampla utilização das tecnologias da informação e comunicação - TIC ${ }^{1}$, dos ambientes virtuais de aprendizagem e mais recentemente, no contexto da Educação Aberta, novas possibilidades podem ser agregadas à sala de aula tradicional com a possibilidade de ampliação da interação professor-aluno-conteúdo. As TIC impactam diretamente o processo de ensino-aprendizagem, alterando a compreensão de tempo e espaço que a sala de aula tem oferecido.

Em um mundo tecnológico como o atual, é impossível pensar a formação universitária distante dos recursos tecnológicos. Assim, várias iniciativas estão voltadas para o preparo dos professores não só para o uso dos instrumentos tecnológicos como instrumentos de ensino, mas também para orientar os estudantes para que se apropriem dos conhecimentos e habilidades necessárias ao uso das novas tecnologias em seu processo de aprendizagem (Andrade \& Vianna, 2015).

No que se refere à Educação Aberta², destacam-se os MOOC, acrônimo de Massive Open Online Course (Cursos On-line Abertos e Massivos), que são capazes de levar conteúdos e aulas de nível universitário com qualidade a qualquer lugar, além de fomentar uma aprendizagem em rede com o foco em processos colaborativos de aprendizagem. Nesse sentido, se faz necessário identificar os estudos sobre a aplicação dos MOOC no contexto do Ensino Superior.

Para problematizar tal afirmativa, em levantamento preliminar realizado em outubro de 2015 em três repositórios distintos, foram identificados 102 trabalhos acadêmicos (teses e dissertações, artigos revisados por pares e trabalhos apresentados em congresso internacional) entre os anos 2011 e 2015, conforme a Tabela 1 a seguir.

1 A terminologia tecnologias de informação e comunicação (TIC)envolve especificamente a aquisição, o armazenamento, o processamento e a distribuição da informação por meios eletrônicos e digitais, como rádio, televisão, telefone e computadores, entre outros. Resultou da fusão das tecnologias de informação, antes referenciadas como informática, e as tecnologias de comunicação, relativas às telecomunicações e mídia eletrônica (Pretto, 2008).

2 Santos (2012) destaca que o termo "Educação Aberta" é utilizado atualmente no contexto dos chamados recursos educacionais abertos (REA), trazendo consigo uma gama de novas práticas de ensino-aprendizagem que se popularizaram com o advento das tecnologias educacionais. 
Tabela 1: MOOC: Pesquisa preliminar em repositórios distintos

\begin{tabular}{l|l|c|c|c|c|c|c}
\hline Repositório & \multicolumn{1}{|c|}{ Escopo } & 2011 & 2012 & 2013 & 2014 & 2015 & Total \\
\hline BDTD & $\begin{array}{l}\text { Biblioteca Digital Brasileira de } \\
\text { Teses e Dissertações - BDTD } \\
\text { (bdtd.ibict.br) }\end{array}$ & 0 & 1 & 3 & 0 & 1 & 5 \\
\hline TISE & $\begin{array}{l}\text { Anais do Congresso Internacional } \\
\text { de Informática Educativa } \\
\text { (www.tise.cl) }\end{array}$ & 0 & 0 & 2 & 4 & 2 & 8 \\
\hline Portal Capes & $\begin{array}{l}\text { Reúne e disponibiliza produção } \\
\text { científica nacional e internacional } \\
\text { (periodicos.capes.gov.br) }\end{array}$ & 0 & 2 & 18 & 37 & 32 & 89 \\
\hline
\end{tabular}

Os resultados obtidos transitaram em variadas áreas, como: Matemática, Ciência da Computação e Engenharias, dentre outras. Evidenciam que a literatura sobre os MOOCs ainda é escassa. As referências relativas aos métodos e às relações de ensino e aprendizagem são incipientes. Percebe-se também a ausência de estudos que tragam uma postura crítica coerente sobre suas potencialidades, aplicações e limitações, verificando-se, portanto, uma oportunidade de explorar essa lacuna.

Diante do exposto, este trabalho tem por objetivo identificar, mapear e analisar os estudos sobre a aplicação dos MOOCs no contexto do Ensino Superior.

Para atingir o objetivo descrito, abordam-se, na seção 2, os conceitos, características fundamentais e tipologias dos MOOC. Na seção 3 são descritos os procedimentos metodológicos que nortearam a condução deste estudo, bem como as fases da pesquisa; na seção 4 são categorizados e analisados os resultados obtidos. Finalizando, na seção 5, são tecidas algumas considerações sobre o panorama da aplicação dos MOOC no Ensino Superior.

Ressalta-se que são identificados na literatura termos como “MOOCs”, “Cursos massivos”, “Massive Open Online Course", "Cursos on-line abertos e massivos", tratados, na maioria das vezes, como sinônimos. Para este estudo, adota-se o termo MOOC (ou MOOCs para denotar plural) como uma expressão equivalente às demais para fins de padronização terminológica.

\section{Conceituando os massive open online courses - MOOC}

Os cursos on-line abertos e massivos, do inglês, são considerados um tipo de curso aberto ofertado por meio de ambientes virtuais de aprendizagem, ferramentas da Web 2.0 ou redes sociais que visam oferecer para um grande número de alunos a oportunidade de ampliar seus conhecimentos dentro de um processo colaborativo.

O termo MOOC foi utilizado pela primeira vez em 2007 por Dave Cormier e Bryan Alexander para definir o curso on-line aberto Conectivismo e Conhecimento Conjuntivo, desenvolvido na Universidade de Manitoba (Canadá) por George Siemens e Stephen Downes.Yuan\& Powell (2013) destacam que

a essência dos MOOC é o espírito da colaboração: além de utilizar conteúdo já disponível gratuitamente na web, boa parte é produzida, remixada e compartilhada por seus participantes durante o próprio curso, em posts, blogs ou fóruns de discussão, recursos visuais, áudios e vídeos, dentre outros formatos (p. 5). 
O objetivo original desta modalidade de curso era proporcionar o acesso gratuito à educação de nível universitário para tantos alunos quanto possível. Eles podem ser vistos como uma extensão de abordagens de aprendizagem on-line existentes e oferecem uma oportunidade para (re)pensar novos modelos de negócios que incluem elementos de educação aberta.

Em contraste com cursos on-line tradicionais, os MOOC têm duas características principais, segundo Butcher (2012):

- Acesso aberto - qualquer um pode participar de um curso on-line. Devem ser gratuitos e abertos para todos os interessados; e

- Escalabilidade - cursos são projetados para suportar um número indefinido de participantes.

Em relação a essas características, apesar de ser aberto, não exigindo pré-requisitos específicos para a participação dos interessados, é fundamental que os participantes tenham pelo menos fluência tecnológica para participar de um MOOC e acesso a um computador ou dispositivo móvel conectados à internet. $\mathrm{E}$, no que se refere à escalabilidade, tal como é proposta, as plataformas que comportam MOOC devem ser projetadas para atender ao aumento exponencial alunos, podendo chegar a centenas de milhares em cada oferta de curso.

Anderson (2013) identifica ainda seis princípios e características, conforme esquema a seguir:

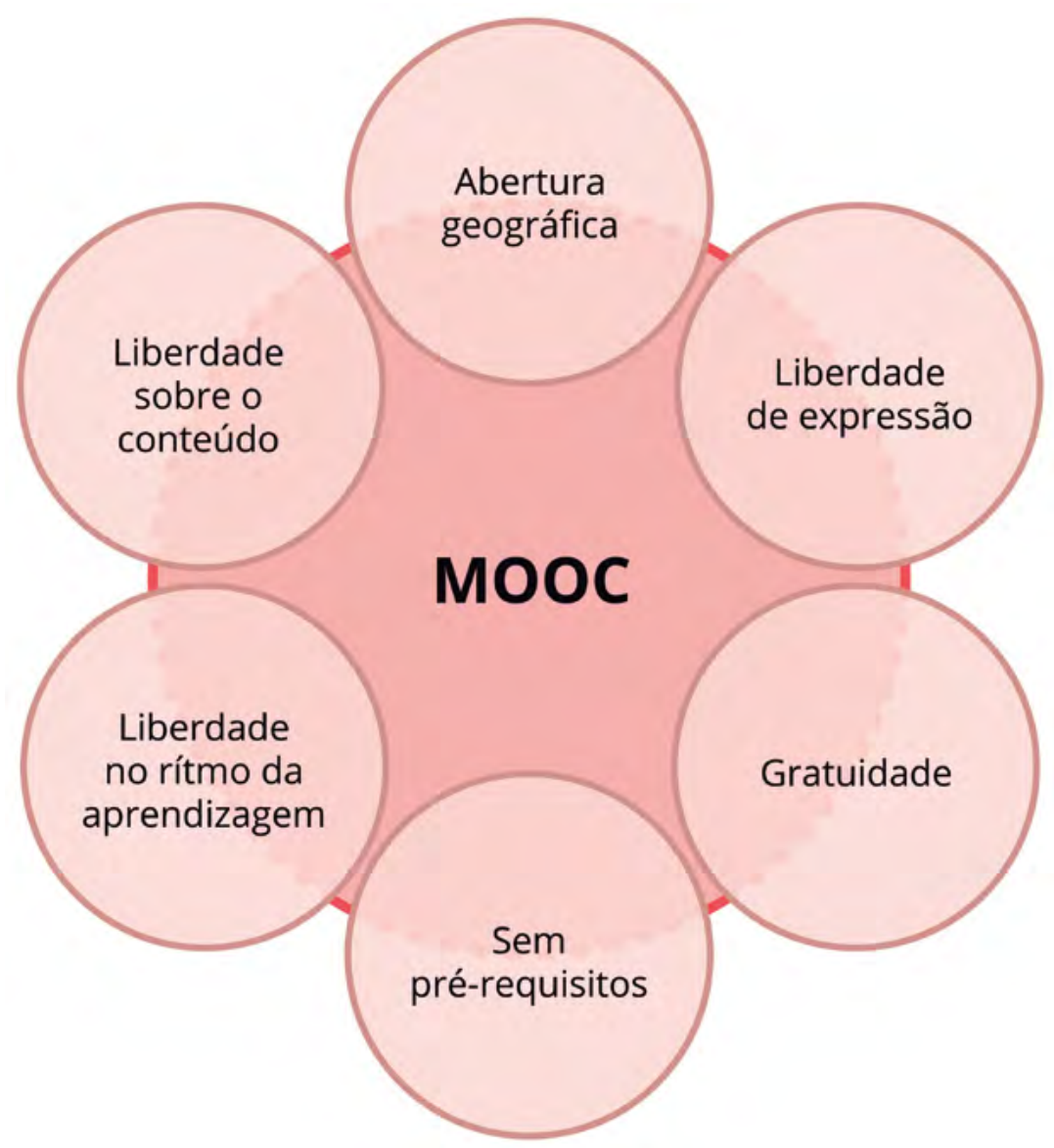

Figura 1: Princípios e características dos MOOC. Adaptado de Anderson (2013).

Dessa maneira, entende-se que os MOOCs podem incentivar a abertura, em termos de custo, de uso de material didático e abertura em relação à construção coletiva do conhecimento. O foco da aprendizagem passa ser o contexto e não apenas o conteúdo. 
Wiley \& Hilton (2009) ressaltam, porém, que essas características podem ser interpretadas de maneira ambígua, variando, inclusive, entre as instituições que ofertam esses cursos. Alguns podem ser massivos, mas não abertos, por exemplo. Isso suscita discussões em torno do licenciamento e das permissões de uso e reúso defendidos pelo movimento REA.

\subsection{Abordagens pedagógicas}

As áreas de pesquisa e experimentação sobre os MOOCs evoluem paralelamente à evolução das ferramentas da Web 2.0, oferecendo novas oportunidades de aprendizagem aos indivíduos. A aprendizagem colaborativa e a alta interatividade entre os participantes podem agregar um grande potencial para o desenvolvimento de aplicações para esses cursos, fomentando, inclusive, a criação novos métodos e modelos de ensino e aprendizagem.

Na literatura, percebem-se várias formas de categorização dos MOOC. A mais conhecida parte do conceito de "abordagens pedagógicas"; pode ser observada na Tabela 2.

Tabela 2: Tipos de MOOC segundo a abordagem pedagógica

\begin{tabular}{l|l}
\hline \multicolumn{1}{c|}{ Categoria } & \multicolumn{1}{c}{ Característica } \\
\hline cMOOC & $\begin{array}{l}\text { Baseado no conectivismo e na interação entre os participantes, acumula } \\
\text { conhecimentos fazendo uso das conexões e links que constrói, dentro da } \\
\text { esfera de interação social. }\end{array}$ \\
\hline xMOOC & $\begin{array}{l}\text { Com fundamento instrucionista, tende a empregar um modelo de trans- } \\
\text { missão do conhecimento, por meio de gravações em vídeo de palestras, } \\
\text { aulas e demais produções audiovisuais; pode incluir exercícios focados } \\
\text { na memorização e reprodução. }\end{array}$ \\
\hline
\end{tabular}

Fonte: Adaptado de Hayes, 2015

Muito embora os dois tipos de MOOC compartilhem características comuns, eles diferem claramente no que diz respeito às teorias de aprendizagem e, em particular, aos modelos pedagógicos. Há também diferenças na maneira como a interação aluno-professor-conteúdo acontece durante os cursos.

Enquanto os cMOOCs são fundamentados no conectivismo ${ }^{3}$, tendo como foco a promoção de oportunidades de aprendizagem colaborativa, e trazem uma proposta pedagógica centrada nos alunos, os xMOOCs seguem um modelo de aprendizagem baseado em transmissão de conteúdos fundamentados em abordagens instrucionistas "enriquecidas" com tecnologias.

O conceito de abertura também tem conotações diferentes; a autonomia do aluno na construção do seu processo de aprendizagem e o uso de redes sociais e interação com os demais alunos são enfatizadas pelos CMOOCs, ao passo que os XMOOCs são baseados em um modelo de tutorias que estabelece uma relação de um-para-muitos para chegar ao conceito de massividade. Ainda em relação aos xMOOCs, ser "aberto" pode significar "sem custo" - com opção de pagamento pela certificação -, não estando, portanto, relacionado com acesso aberto e irrestrito ao conteúdo.

Poy (2015), contudo, pondera e traz algumas críticas no que se refere ao êxito dos MOOCs. Destaca estudos sobre as abordagens e aplicações pedagógicas que precisam de estudos mais detalhados. Ressalta que os cMOOCs necessitam agregar elementos que motivem os alunos afim de incrementar a participação, a interação e a colaboração, além de reduzir a evasão, enquanto para os xMOOCs a interação entre

3 O conectivismo (Siemens, 2004) tem o propósito de responder às novas realidades advindas dos avanços tecnológicos, à Web 2.0 e às transformações econômicas, sociais e culturais, em que os papéis mudam e o aluno deverá ser capaz de encontrar e aplicar o conhecimento quando e onde for necessário. 
os participantes é incipiente e o foco dos cursos fica apenas no conteúdo com avaliação, na maioria das vezes, "tradicional".

\subsection{Desenvolvimento e evolução dos MOOCs}

Como ilustrado na Figura 1, percebe-se um desenvolvimento e evolução constantes do movimento de Educação Aberta e o surgimento do primeiro MOOC em 2008. A partir de então, várias plataformas de aprendizagem foram criadas por instituições de Ensino Superior. O Massachusetts Institute of Technology criou o OpenCourseWare em 2002 e a Open University com aOpenLearns em 2006, são exemplos do interesse e do desenvolvimento contínuo dessas plataformas.

Nota-se que os MOOCs têm atraido atenção de diversas instituições de ensino, inclusive no Brasil, fomentando investimentos e estudos sobre a viabilidade da aprendizagem on-line.

Scortegagna \& Da Silveira (2014) demonstram que, no Brasil, uma das primeiras iniciativas de oferta de MOOC aconteceu em 2012, pela Universidade Estadual Paulista, ao lançar o Portal UNESP Aberta (http:// www.unesp.br/unespaberta). No ano seguinte, oportal brasileiro Veduca lançou o MBA Engenharia e Inovação, em parceria com o UniSEB, sendo considerado o primeiro curso de especialização aberto on-line do mundo, disponibilizando conteúdo em videoaulas e podendo ser acessado gratuitamente por qualquer interessado.

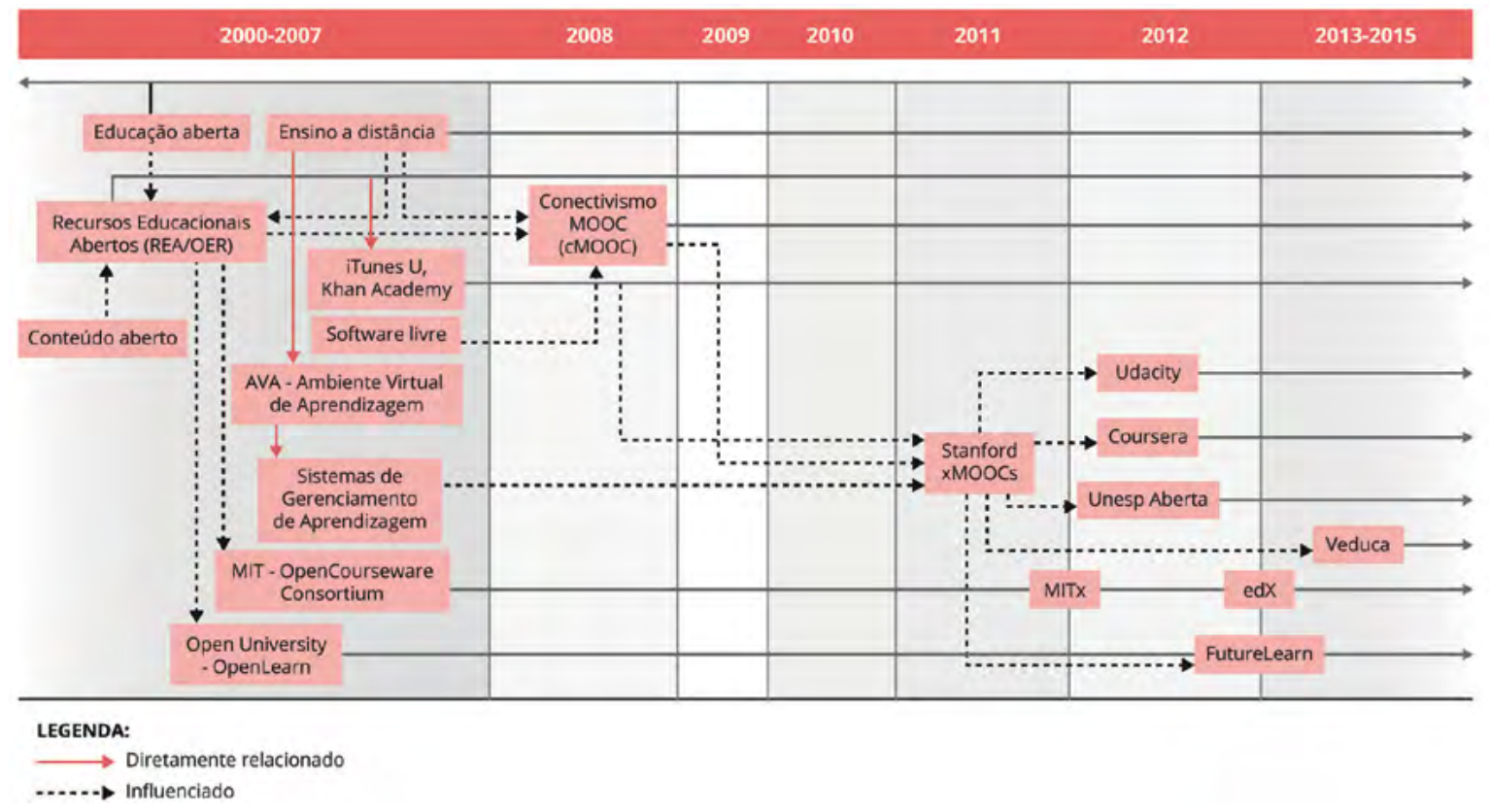

Figura 2: MOOC: a linha do tempo. Adaptado de Yuan \& Powell (2013).

Dentre as várias possibilidades, os MOOCs podem servir de apoio e complemento às aulas tradicionais, nivelamento acadêmico, educação corporativa e processos de formação continuada e de desenvolvimento institucionais. Algumas iniciativas também estão voltadas para a formação continuada de professores.

Bastos \& Biagiotti (2014) enfatizam que as plataformas que mais se destacam na oferta de MOOC são EDX (www.edx.org), Udacity (www.udacity.com) e Coursera (pt.coursera.org). Ressaltam ainda que "apesar de a maioria dos cursos exigirem proficiência em inglês, os países emergentes correspondem a 40\% da audiência dos MOOC". O Brasil já corresponde a 5\% dos usuários do Coursera, atrás apenas dos EUA (27\%) e da Índia (9\%). 


\section{Princípios metodológicos}

Este trabalho se caracteriza como uma pesquisa do tipo "estado da arte", que visa identificar, mapear e analisar os trabalhos sobre a utilização dos MOOCs no Ensino Superior.

Romanowski \& Ens (2006) destacam que as pesquisas do tipo estado da arte

são justificadas por possibilitar uma visão geral do que vem sendo produzido na área e uma ordenação que permite aos interessados perceber a evolução das pesquisas na área, bem como suas características e foco, além de identificar as lacunas ainda existentes (p. 41).

Para tanto, os procedimentos metodológicos foram organizados em quatro etapas:

- Identificação do repositório de informação;

- Definição das estratégias de busca e recuperação da informação;

- Classificação e extração dos dados; e

- Análise e categorização dos artigos.

Na primeira etapa, foi feita a opção pelo Portal de Periódicos da CAPES como principal (e único) repositório de informações, pois este se caracteriza como uma biblioteca virtual que congrega a produção científica nacional e internacional. Disponibiliza um acervo de mais de 37 mil títulos com texto completo, 126 bases referenciais e 11 bases dedicadas exclusivamente a patentes, além de livros, enciclopédias e obras de referência, normas técnicas, estatísticas e conteúdo audiovisual 4.

\subsection{Estratégias de busca e recuperação da informação}

Para selecionar os artigos científicos, utilizaram-se, como estratégia de busca,os descritores "MOOC" or (ou) "Massive Open Online Courses" and (e) "Higher Education", na interface de busca avançada do Portal Capes, tendo como recorte temporal o período de 2011 a 2015.

A busca foi direcionada para qualquer campo (todos os campos) que contivessem os termos destacados acima.

4 Portal de Periódicos: missão e objetivos. Disponível em: www.periodicos.capes.gov.br. Acesso em: 01 dez. 2015. 

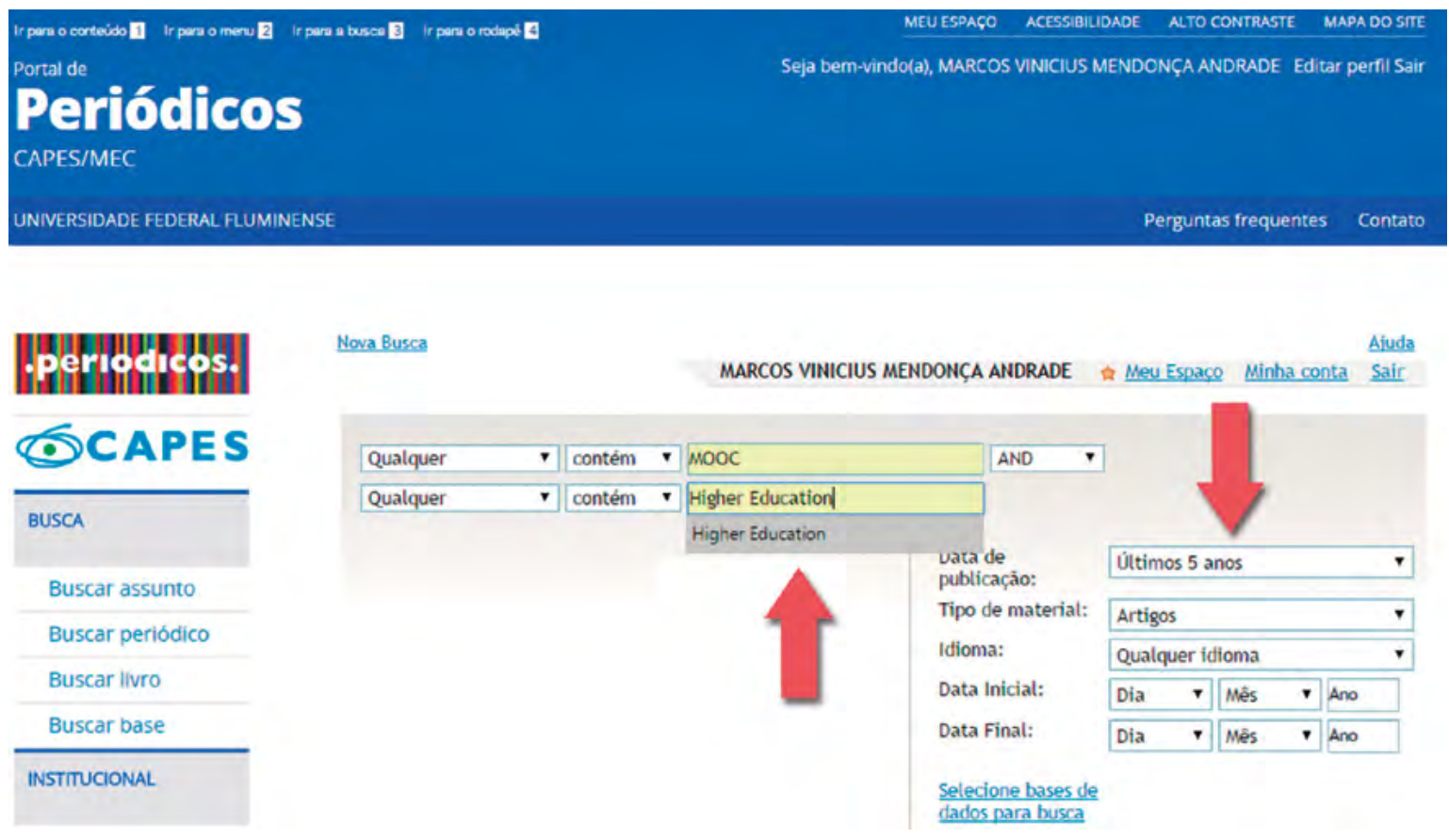

Figura 3: Modelo da interface de busca avançada do Portal Capes.

É importante ressaltar que se optou pelos descritores na língua inglesa, uma vez que a grande maioria das bases de dados que integram o Portal de Periódicos da Capes são internacionais. Inclusive o próprio Portal sugere que sejam utilizados termos em inglês, considerando que a literatura científica é, em sua maioria, publicada em inglês, aumentando a recuperação da informação. Não há, entretanto, restrição a outros idiomas.

As estratégias de busca adotadas na fase inicial deste mapeamento são fundamentais para o andamento da pesquisa, considerando que uma estratégia inconsistente pode trazer um grande número de trabalhos e possivelmente a pesquisa poderia ter outro direcionamento.

Os critérios utilizados para a seleção dos artigos permitiram refinar a busca e recuperar os estudos relevantes e pertinentes ao objeto da pesquisa, sendo descartados os demais itens coletados. Não foram observados itens duplicados.

\subsection{Classificação e extração dos dados}

Neste primeiro recorte, foram obtidos 351 artigos, distribuídos em 12 bases de dados. Em seguida, novos filtros e critérios de busca foram adotados para aumentar a precisão e a granularidade dos resultados. Para tal, foram selecionados os artigos revisados por pares, escritos em português, inglês ou espanhol que estivessem com os textos completos disponíveis, chegando ao quantitativo de 24 artigos, conforme ilustrado no Gráfico 1, a seguir: 


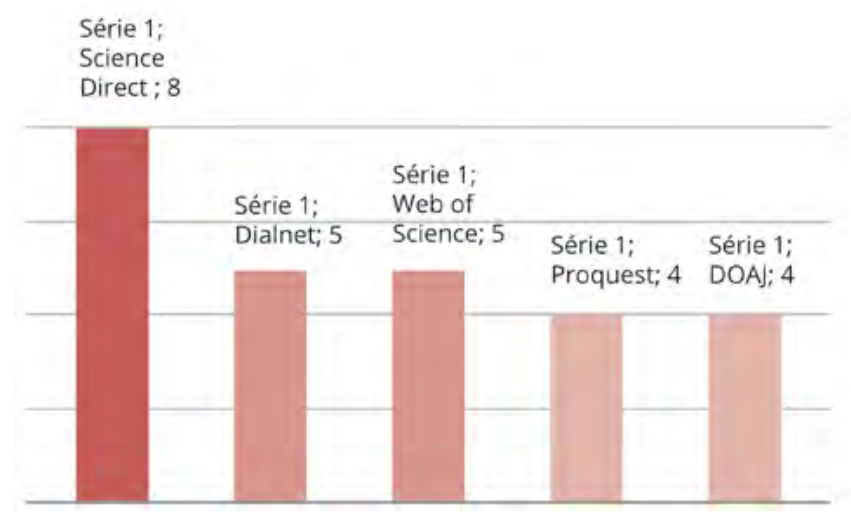

Gráfico 1: Recuperação da informação das publicações por base de dados (nov/2015)

A base de dados Science Direct apresentou o resultado mais significativo (31\%) em relação à quantidade de artigos recuperados, seguida das bases Web of Science (19\%) e Dialnet (19\%).

É importante destacar que a plataforma ScienceDirect (www.sciencedirect.com) disponibiliza trabalhos completos, tendo todo o conteúdo revisado por pares (sendo este, inclusive, um dos critérios da pesquisa), agregando atualmente $25 \%$ da produção científica atual.

\section{Análise e categorização dos artigos}

Inicialmente não foi observado um padrão de recorrência em relação aos autores que mais publicam sobre a temática. No que se refere aos periódicos, foram identificados 16 títulos, em quese destacam International Review of Research in Open and Distance Learning, com 17\% dos artigos, International Journal of Information and Education Technology e Profesorado com 13\% das publicações cada um.

\subsection{Distribuiç̧ão dos artigos por ano de publicação}

No que se refere ao ano de publicação, evidenciam-se os seguintes dados apresentados no Gráfico 2, a seguir:

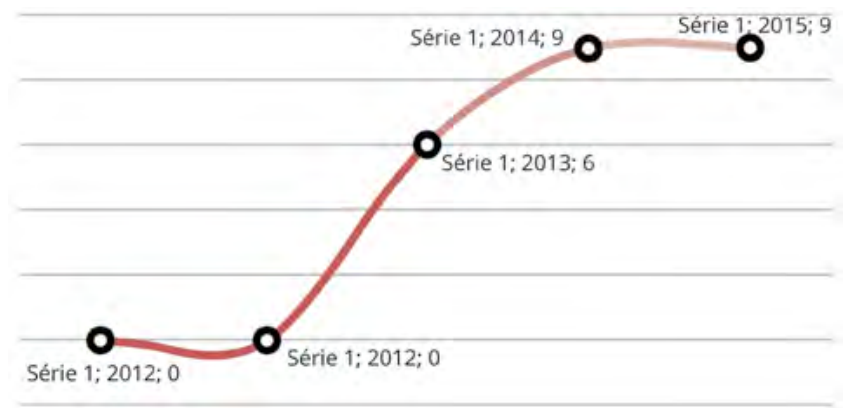

Gráfico 2: Distribuição dos artigos por ano de publicação.

Há concentração nos últimos três anos, tendo padrão de crescimento. Tal comportamento talvez se justifique em função de os MOOCs no Ensino Superior serem considerados "tecnologia emergente", de acordo com o relatório Technologies in Higher Education: Mapping the Terrain ${ }^{5}$, publicado pela Unesco em 2014.

O incremento das pesquisas nos últimos três anos procura obter melhor compreensão do fenômeno

5 UNESCO. Institute for Information Technologies in Education. Technologies in Higher Education: Mapping the Terrain. Disponível em: $\underline{\text { http://iite.unesco.org/pics/publications/en/files/3214737.pdf }}$ 
dos MOOCs e de seu potencial de inovação como parte da tendência para maior abertura no Ensino Superior. Esses cursos podem trazer oportunidades para a expansão do acesso ao Ensino Superior, além de criar um espaço para experimentações de novos modelos de ensino e aprendizagem on-line (Poy, 2014).

\subsection{Distribuição por assuntos e palavras-chave}

No conjunto dos artigos selecionados, foram identificadas 44 palavras-chave. Essas palavras-chave foram informadas pelos autores e indexadas pelas bases de dados que compõem o Portal de Periódicos.

Para representar o conjunto de palavras utilizou-se a ferramenta TagCroud (www.tagcroud.com) para gerar uma nuvem de palavras. Os termos com maior destaque são os que mais se repetem como palavras-chave na lista, conforme ilustrado na figura a seguir.

\section{access adaptive analysis based business doud content COUrSe criteria cultural distance distancedication e-learning education

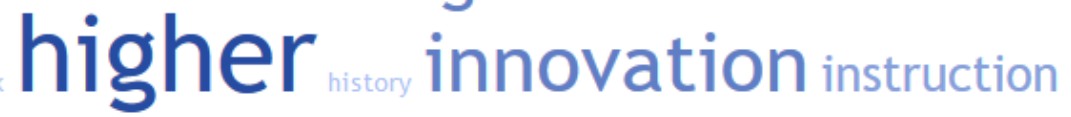 oer online open partnerships ${ }_{a r}$ quality reform stategeses teaching technology}

Figura 3: Nuvem de palavras

Dentro do esperado, os termos e-learning e education higher se destacam, levando em consideração a escolha dos termos utilizados no processo de pesquisa no Portal de Periódicos. Os termos teaching (docência) e inovação também ganham destaque.

\subsection{Categorização por temas}

Após a leitura dos resumos e, em alguns casos, dos artigos na íntegra, delineou-se uma categorização a partir da sistematização dos dados coletados, conforme demonstrado na Tabela 3.

Evidencia-se que os trabalhos ainda estão muito focados, $48 \%$ do total, nas plataformas e nos ambientes virtuais de aprendizagem que disponibilizam os MOOCs. As mais citadas são Coursera, Udacity e MiriadaX, todas com fins lucrativos. Essas plataformas, desenvolvidas em grande parte de forma independente das instituições de ensino, ofertam inúmeros cursos "gratuitos". As plataformas têm como fontes principais de receita a cobrança pela certificação, patrocínios e anúncios diversos.

Alguns estudos sugerem que as instituições de ensino têm observado o crescimento da oferta dos MOOCs e já analisam formas de incorporá-los ao contexto acadêmico, gerando inclusive novas oportunidades de negócios. Essas instituições estão estudando os modelos pedagógicos mais adequados e inovadores no contexto do Ensino Superior. Outra grande preocupação está focada nos critérios de qualidade dos MOOCs e na grande evasão dos alunos em relação a esta modalidade de curso.Também não são evidentes as estratégias de aplicação dos MOOCs para servir, por exemplo, aos alunos com necessidade de aprendizagens mais complexas. 
Tabela 3: MOOC e Ensino Superior - categorização por temas

\begin{tabular}{|c|c|c|c|c|}
\hline Temática principal & $\begin{array}{l}\mathrm{N}^{\circ} \text { de } \\
\text { artigos }\end{array}$ & $\%$ & Subtemas & $\begin{array}{l}\mathrm{N}^{\circ} \text { de } \\
\text { artigos }\end{array}$ \\
\hline \multirow{2}{*}{ Objetos de aprendizagem } & \multirow{2}{*}{2} & \multirow{2}{*}{9} & Acessibilidade & 1 \\
\hline & & & Reúso e direito autoral & 1 \\
\hline \multirow{3}{*}{ Docência } & \multirow{3}{*}{5} & \multirow{3}{*}{21} & Ensino-aprendizagem & 1 \\
\hline & & & Formação continuada & 3 \\
\hline & & & Educação on-line & 1 \\
\hline \multirow{2}{*}{$\begin{array}{l}\text { Ambientes virtuais de } \\
\text { aprendizagem }\end{array}$} & \multirow{2}{*}{10} & \multirow{2}{*}{42} & Tipos e plataformas & 6 \\
\hline & & & Modelos de ensino & 4 \\
\hline \multirow{3}{*}{ Instituições de Ensino Superior } & \multirow{3}{*}{9} & \multirow{3}{*}{38} & Estratégias mercadológicas & 5 \\
\hline & & & Processo de inovação & 2 \\
\hline & & & Controle da qualidade & 2 \\
\hline Totais & 24 & 100 & & 24 \\
\hline
\end{tabular}

No que se refere aos modelos pedagógicos, os estudos estão mais direcionados para a criação de ambientes de aprendizagem eficazes, fato que traz a impressão de que o ensino mediado por um MOOC se reduz ao design do ambiente educacional e tecnológico e à sua manipulação. As responsabilidades dos docentes parecem ser reduzidas à facilitação do ambiente.

As pesquisas em relação à docência (21\%) apontam a necessidade de formação continuada para os professores, que necessariamente passarão a produzir conteúdos, adotar novas formas de interação com os alunos e com as instituições de ensino. Como serão as relações de trabalho, caso os MOOCs venham a se estabelecer como um modelo de negócio? Com os MOOCs estabelecendo um modelo "massivo", sem limites de alunos por curso, como será fomentada a relação professor-aluno? São algumas questões que precisam de estudos mais aprofundados.

Na categoria Educação Aberta estão o "aprendizado e pesquisa em qualquer suporte ou mídia que estão sob domínio público ou estão licenciados de maneira aberta, permitindo que sejam utilizados ou adaptados por terceiros" (Santos, 2015). Logo, ainda há algumas lacunas em relação aos direitos autorais e às permissões para reúso, remixagem e redistribuição de conteúdos e de financiamento por parte das instituições de ensino, sobretudo aquelas com fins lucrativos.

\section{Considerações finais}

Neste trabalho foi realizado um mapeamento sistemático para identificar os estudos sobre a aplicação dos MOOCs - Massive Open Online Course - no contexto do Ensino Superior. Inicialmente, são percebidas evidências de crescente interesse por essa modalidade de curso e a literatura revisada por pares está em ascensão, mas ainda limitada.

Pode-se verificar que a grande maioria dos trabalhos está relacionada com as categorias Ambientes virtuais de aprendizagem e Instituições de Ensino Superior, que veem nos MOOCs uma oportunidade, um novo modelo de negócios. Entretanto, alguns estudos afirmam que a viabilidade econômica dos MOOCs ainda é um ponto de incerteza. 
Poucos são os estudos relacionados ao papel do professor diante desse novo cenário de aprendizagem. A elaboração e a apropriação de conteúdos também carecem de estudos, uma vez que o público-alvo dos MOOCs é muito diversificado.

Percebeu-se que, em se tratando de Brasil, ainda não há estudos aprofundados sobre os MOOCs no âmbito do Ensino Superior. Algumas iniciativas apontam o uso dos MOOCs como estratégia de reforço para conteúdos no Ensino Médio, mas as publicações ainda são muito incipientes.

Com o mapeamento realizado, foi possível verificar a aplicação dos MOOCs no contexto do Ensino Superior. Pode-se ainda constatar que este é um tema emergente e necessita ser mais explorado. Essa modalidade de curso pode trazer significativos avanços - e desafios -, tanto nos ambientes formais quanto informais de aprendizagem.

\section{Referências}

Anderson, T. (2015). MOOCs and open and distance learning. Disponível em: http://www.ethicalforum.be/ sites/default/files/MOOCsPromisePeril.pdf

Andrade, M. V. M. \& Vianna, A. A. (2015). Ambiente de educação a distância direcionado à formação continuada de professores universitários: um estudo de caso. Nuevas Ideas en Informática Educativa. TISE, 2015. Disponível em: http://www.tise.cl/volumen11/TISE2015/774-779.pdf

Barín, C., S. \& Bastos, F. P. da (2013). Problematização dos MOOCs na atualidade: Potencialidades e Desafios. Renote, 11(3). Disponível em: seer.ufrgs.br/index.php/renote/article/view/44707

Bastos, R. C. \& Biagiotti, B. (2014). MOOCs: uma alternativa para a democratização do ensino. Renote, 12(1). Disponível em: seer.ufrgs.br/index.php/renote/article/view/50333/31417

Butcher, N.(2014). Technologies in Higher Education: mapping the terrain. New York: Unesco, 2014. Disponível em: iite.unesco.org/pics/publications/en/files/3214737.pdf

Haguenauer, C. J., Mussi, M. V. \& Cordeiro Filho, F. (2009). Ambientes virtuais de aprendizagem: definições e singularidades. Educaonline, 3(2), maio/ago. 2009.

Hayes, S. (2015). MOOCs and quality: a review of the recent literature. Gloucester: QAA.

Kuntz, V. H. \& Ulbricht, V. R. (2014). Panorama dos estudos de usabilidade em Massive Open Online Course (MOOC). Nuevas Ideas en Informática Educativa. TISE, 2014. Disponível em: tise.cl/2015/img/ TISE2014.pdf

Poy, Raquel. (2014). Factores de exito de los MOOC: algunas consideraciones criticas. RISTI: Revista Ibérica de Sistemas e Tecnologias de Informação, 14.

Pretto, N. L. (2008). Além das redes de colaboração: internet, diversidade cultural e tecnologias do poder. Salvador: EDUFBA.

Romanowski, J. P. \& Ens, R. T.(2006). As pesquisas denominadas do tipo "estado da arte" em educação. Diálogo Educ., Curitiba, 6(19), pp. 37-50. Disponível em: www.scielo.br/pdf/es/v23n79/10857.pdf

Santos, A. I. (2015). Educação aberta: histórico, práticas e o contexto dos recursos educacionais abertos. In: Santana, B.; Rossini, C.; Pretto, N. L. (org.). Recursos educacionais abertos: práticas colaborativas, políticas públicas. (pp. 71-89). Salvador: EDUFBA.

Scortegagna, L. \& Silveira, L. F.(2014). Massive Open Online Course (MOOC) na Educação Matemática: Possibilidades. Atas do XXV Seminário de Investigação em Educação Matemática. (pp. 449-452).Braga: APM. 
Siemens, G. (2004). Connectivism: a learning theory for the digital age. Disponível em: www.elearnspace. org/Articles/connectivism.htm

Yuan, L. \& Powell, S. (2013). MOOCs and Open Education: implications for Higher Education. London: CETIS. Disponível em: http://publications.cetis.org.uk/wp-content/uploads/2013/03/MOOCs-and-Open-Education.pdf

Wiley, D. \& Hilton, J. Openness, dynamic specialization, and the disaggregated future of Higher Education. IRRODL, 10(5). Disponível em: http://www.irrodl.org/index.php/irrodl/article/view/768/1414

\section{ANEXO I - Relação de artigos analisados}

[1] Aaron, Lynn S.; Roche, Catherine M. Intellectual Property Rights of Faculty in the Digital Age - Evolution or Dissolution in 21st Century Academia? Journal of Educational Technology Systems, v. 43, n. 3, p.320-341.

[2] Admiraal, Wilfried; Huisman, Bart;Pilli, Olga. Assessment in massive open online courses. Electronic Journal of e-Learning, v. 13, n. 4, 2015, p.207-216.

[3] Aguaded Gómez, José Ignacio; Medina Salguero, Rosario. Criterios de calidad para la valoración y gestión de MOOC. RIED: Revista Iberoamericana de Educación a Distancia, v. 18, n. 2, 2015, p.119-143.

[4] Berrocoso, Jesús Valverde. MOOCS: A critical view from the Education Sciences. Profesorado, v. 18, n. 1, jan-abr, 2014, p.93-111.

[5] Bulfin, Scott; Pangrazio, Luci;Selwyn, Neil. Making 'MOOCs': The construction of a new digital higher education within news media discourse. International Review of Research in Open and Distance Learning, v. 15 , n. 5, 2014, p.290-305.

[6] Daniel, John; Cano, Esteban Vázquez; Gisbert Cervera, Mercè. The future of MOOCs: Adaptive learning or business model? RUSC Universities and Knowledge Society Journal, v. 12, n. 1, jan. 2015. p.64-73.

[7] Dyer, Ronald A.D. Exploring the Relevancy of Massive Open Online Courses (MOOCs): A Caribbean University Approach. Information Resources Management Journal, v. 27, n. 2, abr. 2-14. p.61-77.

[8] El Ahrache, Sara Ibn; Badir, Hassan Badir; Tabaa, Yassine; Medouri, Abdellatif. Massive Open Online Courses: a new dawn for Higher Education? International Journal on Computer Science and Engineering, v. 5, n. 1, maio 2013. p. 323-327.

[9] Fisher, William W. HLS1X: CopyrightX, Spring 2013, Course Report. HarvardX Working Paper Series, v. 5, .1, 2013, p. 1-31.

[10] Galán, Jose Gómez. The MOOC phenomenon and universality of culture. The news boundaries of higher education, v. 18, n. 1, jan-abr., 2014. p.73-91.

[11] Gasevic, D.; Kovanovic, V.;joksimovic, S.; Siemens, G. Where is Research on Massive Open Online Courses Headed? A Data Analysis of the MOOC Research Initiative. International Review Of Research In Open And Distance Learning, v. 5, n. 5, nov. 2014. p.134-176.

[12] Gil-Jaurena, Gil. Openness in higher education. Open Praxis, v. 5, n. 1, jan. 2013, p.1-98.

[13] Graván, Pedro Román; Rey, Juan Manuel Méndez. Educational innovation experience with MOOC's course: QR codes applied to teaching. Profesorado, v. 18, n. 1, jan-abr., 2014. p.113-136.

[14] Heinsch, Bárbara; Rodríguez Pérez, María De Las Nieves. MOOC: un nuevo escenario de enseñanza -aprendizaje de lenguas extranjeras. @tic: Revista d'innovació educativa, n. 14, 2015, p.1-12. 
[15] Kalman, Yoram. A race to the bottom: MOOCs and higher education business models. Open Learning, v. 29, n. 1, jan. 2014, p.5-14.

[16] Lopez-Meneses, E.; Vazquez-Cano, E.; Roman, P. Analysis and Implications of the Impact of MOOC Movement in the Scientific Community: JCR and Scopus (2010-13). Comunicar, v. 44, jan. 2015, p.73-80.

[17] Medina-Salguero, Rosario; Aguaded, Ignacio. The MOOC in the educational platform MiriadaX. Profesorado, v. 18, n. 1, jan-abr., 2014. p.137-153.

[18] Ong, Beng; Grigoryan, Ani. MOOCs and Universities: Competitors or Partners? International Journal of Information and Education Technology, v. 5, n. 5, maio 2015, p. 373-376.

[19] RICHTER, S. Preparing Faculty for Teaching a MOOC: Recommendations from Research and Experience. International Journal of Information and Education Technology, v. 4, n. 5, 2014. p. 411-415.

[20] Sancho-Vinuesa, T.; Oliver, M.;Gisbert, M. MOOCS in Catalonia: fueling innovation in higher education. Educacion XX1, v. 18, n. 2, 2015, p.125-146.

[21] Sangra, A.; Gonzalez-Sanmamed, M.;Anderson, T. Meta-analysis of research on MOOC along the period 2013-2014. Educacion XX1, v. 18, n. 2, 2015, p.21-49.

[22] Scott Bulfin; Luci Pangrazio; Neil Selwyn. Making 'MOOCs': The Construction of a New Digital Higher Education within News Media Discourse. International Review of Research in Open and Distance Learning, v. 15, n. 5, nov. 2015.

[23] Soffer, Tal; Cohen, Anat. Implementation of Tel Aviv university MOOCs in academic curriculum: a pilot study. International Review of Research in Open and Distance Learning, v. 16, n. 1, 2015, p.80-97.

[24] Toven-Lindsey, B.; Rhoads, R. A.; Lozano, J. B. Virtually unlimited classrooms: pedagogical practices in massive open online courses. The Internet and Higher Education, v. 24, 2015, p.1-12. 